\title{
Caracterização de Atendimentos em uma Rede de Atenção à Saúde
}

\author{
Leonardo de O. Jasmim ${ }^{1}$, Artur Ziviani ${ }^{1}$, Marcia Ito ${ }^{2}$, Paulo B. Paiva ${ }^{3}$ \\ ${ }^{1}$ Laboratório Nacional de Computação Científica (LNCC) - Petrópolis/RJ - Brasil \\ ${ }^{2}$ IBM Research Brazil - São Paulo/SP - Brasil \\ ${ }^{3}$ Departamento de Informática em Saúde \\ Universidade Federal de São Paulo (UNIFESP) - São Paulo/SP - Brasil
}

\{ljasmim,ziviani\}@lncc.br, marciaito@br.ibm.com, paiva@unifesp.br

\begin{abstract}
Healthcare networks are a complex system. Currently, there is trend towards applying methods from complex network analysis to model and analyze such systems. This approach enables the study of the flow of patients through the care services in order to identify relevant patterns, improve resource allocation, and promote better service to the population. This is an ongoing work in which we present a first exploratory study aiming at characterizing the assistance in a public healthcare network, which is a starting point for a broader study of the patient flows based on the theory of complex networks.
\end{abstract}

Resumo. Redes de atenção à saúde formam um sistema complexo. Atualmente há uma tendência crescente de aplicação de métodos de análise de redes complexas para modelar e analisar tais sistemas. Essa abordagem possibilita o estudo do fluxo de atendimento dos pacientes para a identificação de padrões relevantes, a melhoria na alocação dos recursos e a promoção de melhor serviço à população. Este é um trabalho em andamento no qual apresentamos um primeiro estudo exploratório visando a caracterização de atendimentos em uma rede de atenção à saúde pública, ponto inicial para um estudo mais amplo de fluxo de atendimentos com base na teoria de redes complexas.

\section{Introdução}

A interconectividade dos diversos componentes que englobam as redes de atenção à saúde (unidades de saúde, tipos de serviço prestados, profissionais e pacientes) tipicamente gera um sistema muito complexo, tendo em vista a diversidade e densidade destas interações, dificultando a análise do comportamento desta rede. Nesse contexto, observa-se uma tendência na utilização de técnicas de modelagem e análise de redes complexas na área da saúde, não apenas para modelos de propagação de doenças, mas também as relacionando à organização dos serviços de saúde, visando a melhor coordenação de cuidados e uso de recursos de forma centrada no paciente [Pollack et al. 2013, Ito et al. 2016, Barnett et al. 2011, Findlay and Grant 2011, Thorwarth and Arisha 2012].

Ao fazer uso da rede de atenção à saúde, os pacientes estabelecem fluxos de acesso aos serviços, criando relacionamentos entre os componentes da rede que podem ser empiricamente representados conforme a Figura 1. Analisar os fluxos de atendimento de pacientes com características similares de demanda pode auxiliar os gestores do sistema 


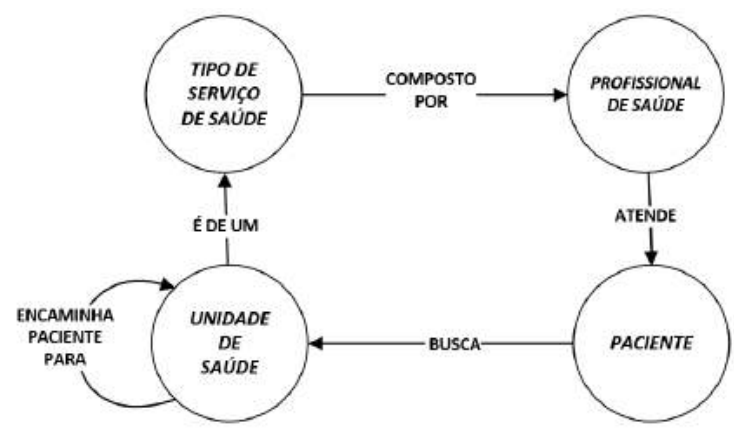

Figura 1. Relacionamento de entidades nas redes de atenção à saúde.

na melhor organização dos recursos, bem como na oferta de um atendimento mais eficiente do ponto de vista do paciente. Em uma primeira etapa do projeto mais amplo de análise do fluxo de atendimentos em serviços de saúde, neste artigo de trabalho em andamento, apresentamos a caracterização de atendimentos em uma rede de atenção à saúde do sistema público de saúde. Com isso, visamos criar subsídios para futuras avaliações baseadas em redes complexas, verificando a dinâmica de redes de atenção à saúde sob novas perspectivas, buscando o melhor entendimento da rede e apoio para a organização de recursos para melhorias da saúde da população atendida.

\section{Descrição e caracterização do conjunto de dados}

Foram avaliados dados referentes a agendamentos e atendimentos do serviço público de saúde da cidade de São Paulo (SP) ${ }^{1}$ apurados durante os anos de 2014 e 2015, distribuídos em três blocos distintos, conforme descrição mostrada na Tabela 1. Inicialmente foram selecionados os atributos relevantes para a caracterização das principais entidades que atuam nesta rede, a saber: Paciente; Unidade de Saúde; Tipo de Serviço; e Profissional de Saúde. Foram avaliados também atributos referentes aos Diagnósticos e Procedimentos, pois estes serão importantes em um futura qualificação dos relacionamentos. Posteriormente, foi verificada a integridade dos registros com relação a tais atributos.

Tabela 1. Descrição dos datasets avaliados.

\begin{tabular}{|l|r|c|c|}
\hline \multicolumn{1}{|c|}{ Dataset } & \# Registros & \# Atributos por registro & \# Registros válidos \\
\hline Agendamento & 6.160 .394 & 24 & $89,6 \%$ \\
\hline Atendimento com diagnóstico & 30.984 .034 & 17 & $98,4 \%$ \\
\hline Atendimento com procedimento & 27.560 .982 & 18 & $100 \%$ \\
\hline
\end{tabular}

Foram identificados 6.567.257 pacientes distintos, o que é primordial para acompanhar o fluxo de atendimentos desses pacientes através dos serviços disponíveis. Nesse sentido, foi avaliada a distribuição dos registros válidos. ${ }^{2}$ Identificamos alguns outliers (Figura 2(a)), porém a maior parte dos pacientes apresenta um comportamento esperado (Figura 2(b)), sinalizando para a integridade do atributos de distinção dos pacientes.

Com relação às unidades de saúde, foram avaliados: nome, tipo e localização. Identificamos 1006 unidades agrupadas em 30 tipos de serviço. Quanto aos agendamentos, são as Unidades Básicas de Saúde (UBS) as maiores solicitantes (85\%), sendo a

\footnotetext{
${ }^{1}$ Utilização dos dados aprovada pelo Comitê de Ética e Pesquisa (CAAE: 51038515.6.3001.0086).
}

${ }^{2}$ Entenda-se por registro válido aquele no qual é possível distinguir unicamente um paciente. 
(a)

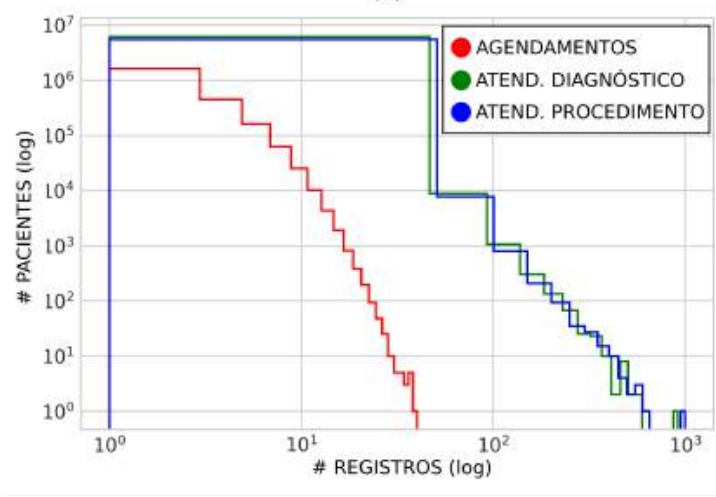

(b)

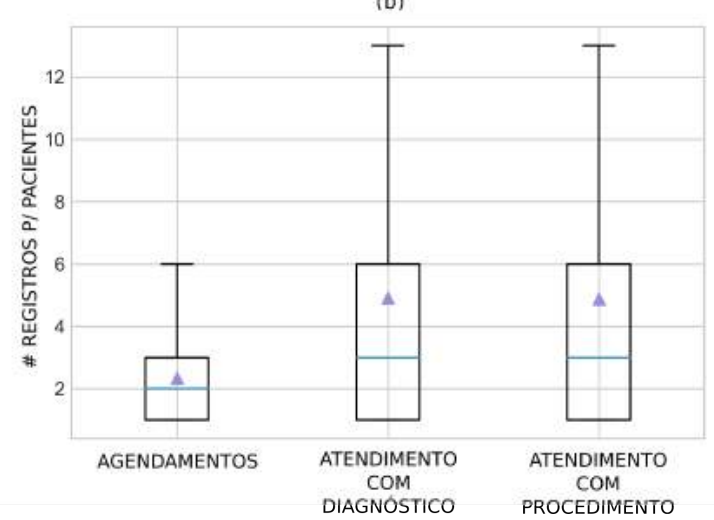

Figura 2. Frequência dos registros e distribuição por pacientes.

maior parte destes (29\%) direcionados aos Ambulatórios de Especialidades, o que pode ser um sinalizador de que o direcionamento do paciente é realizado pela UBS. Verificamos também que algumas poucas unidades concentram um maior número de registros, que pode ser um sinalizador de hubs na rede. O agrupamento da demanda pelo tipo de serviço pode auxiliar na busca de um modelo que venha a refletir tal aspecto. Nos dados observados, os serviços são estruturados em 4 níveis organizacionais, que possibilitará mensurar o fluxo dos paciente em vários níveis de granulosidade espaço-temporal.

Os dados de atendimentos trazem o Código Brasileiro de Ocupação $(\mathrm{CBO})^{3}$ e a descrição da classe dos profissionais de saúde que assistiram o paciente, distribuídos em 152 tipos de profissionais. Apenas $2 \%$ dos registros não tem identificação de CBO. Essa informação irá auxiliar na distinção das unidades que, apesar de serem de um mesmo tipo, podem não ser compostas por equipes similares. A Figura 3 traz os 8 tipos de profissionais que mais prestaram assistência aos pacientes no período avaliado.
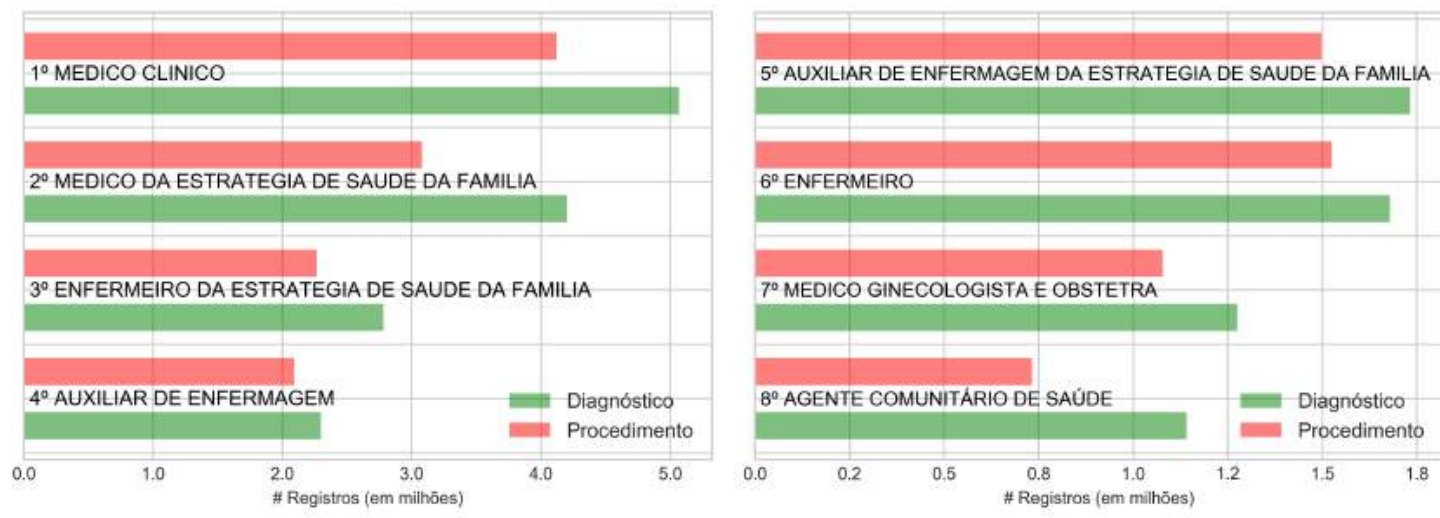

Figura 3. Frequência de atendimentos por tipo de profissional.

Os blocos de registro de atendimentos distinguem-se entre atendimentos com diagnóstico e com procedimento, porém estes tem relação entre si, fato perceptível na Figura 2(a). Futuramente burcar-se-á estabelecer tal relação através dos dados em comum, de forma a consolidar os dados de atendimento em um único bloco de dados.

${ }^{3} \mathrm{CBO}:$ http://www.mtecbo.gov.br/cbosite/pages/home.jsf 
O primeiro bloco apresenta o Código Internacional de Doenças (CID-10) ${ }^{4}$ e a indicação do diagnóstico identificado. Apesar de 83,8\% dos registros não estarem com diagnóstico identificado, a base conta com 9116 diagnósticos distintos e entre os diagnósticos mais frequentes estão Infecção Aguda das Vias Aéreas Superiores (J06.9) e Hipertensão Primária (I10). Para melhorar a classificação, poderão ser utilizados os capítulos da CID-10, gerando melhores agrupamentos sobre o perfil dos pacientes. No outro bloco é disponibilizado nome e código do procedimento realizado, código este referente à tabela do Sistema de Gerenciamento da Tabela de Procedimentos, Medicamentos e OPM do SUS (SIGTAP). ${ }^{5}$ Esse bloco conta com 1000 procedimentos distintos, sendo que 7,84\% destes estão sinalizados com a tag "NÃO SE APLICA" e o mais registrado foi a Consulta Médica em Atenção Primária (03.01.01.0064A). Ambos atributos dão suporte para a definição do perfil do paciente e espera-se que pacientes com perfis semelhantes atravessem fluxos equivalentes. Apesar da má distribuição da amostra com relação a tais pontos, seus códigos permitem que sejam realizadas classificações com maior generalidade e melhor distribuição das amostras, em diferentes níveis.

\section{Considerações finais}

O objetivo de nosso projeto de forma mais ampla é a análise do fluxo de atendimento de pacientes em serviços de saúde com base na teoria de redes complexas. O presente artigo descreve nossos primeiros passos nessa direção, já trazendo como contribuição resultados preliminares acerca da caracterização de atendimentos considerando dados de uma grande e representativa rede de atenção à saúde. Para a continuidade do trabalho, visamos o estudo do fluxo de atendimento dos pacientes para a identificação de padrões relevantes, a melhoria na alocação dos recursos e a promoção de melhor serviço à população.

\section{Referências}

Barnett, M. L., Landon, B. E., O'Malley, A. J., Keating, N. L., and Christakis, N. A. (2011). Mapping physician networks with self-reported and administrative data. $\mathrm{He}$ alth Services Research, 46(5):1592-1609.

Findlay, M. and Grant, H. (2011). An application of discrete-event simulation to an outpatient healthcare clinic with batch arrivals. In Proc. of the IEEE Winter Simulation Conference (WSC), pages 1166-1177.

Ito, M., Moyano, L. G., Appel, A. P., and Santana, V. F. (2016). Análise do relacionamento na comunidade de médicos de seguradoras de saúde. In Anais do XV Congresso Brasileiro de Informática em Saúde (CBIS).

Pollack, C. E., Weissman, G. E., Lemke, K. W., Hussey, P. S., and Weiner, J. P. (2013). Patient sharing among physicians and costs of care: A network analytic approach to care coordination using claims data. Journal of General Internal Medicine, 28(3):459465 .

Thorwarth, M. and Arisha, A. (2012). A simulation-based decision support system to model complex demand driven healthcare facilities. In Proc. of the IEEE Winter Simulation Conference (WSC), page 90.

\footnotetext{
${ }^{4}$ CID-10: http://apps.who.int/classifications/icd10/browse/2016/en

${ }^{5}$ SIGTAP : http:sigtap.datasus.gov.br
} 